\title{
Finite element investigations on the microstructure of fibre-reinforced composites
}

\author{
A. R. Maligno*, N. A. Warrior, A. C. Long \\ School of Mechanical, Materials and Manufacturing Engineering, The University of Nottingham, University Park; \\ Nottingham, NG7 2RD, UK
}

Received 21 June 2008; accepted in revised form 8 August 2008

\begin{abstract}
The effect of residual stress due to the curing process on damage evolution in unidirectional (UD) fibre-reinforced polymer-matrix composites under longitudinal and transverse loading has been investigated using a three-dimensional micromechanical representative volume element (RVE) model with a hexagonal packing geometry and the finite element method. Residual stress has been determined by considering two contributions: volume shrinkage of matrix resin from the crosslink polymerization during isothermal curing and thermal contraction of both resin and fibre as a result of cooling from the curing temperature to room temperature. To examine the effect of residual stress on failure, a study based on different failure criteria and a stiffness degradation technique has been used for damage analysis of the RVE subjected to mechanical loading after curing for a range of fibre volume fractions. Predicted damage initiation and evolution are clearly influenced by the presence of residual stress.
\end{abstract}

Keywords: damage mechanism, residual stress, failure criteria

\section{Introduction}

The fabrication process of fibre-reinforced polymer matrix composite materials requires a high temperature curing procedure. Stresses are generated during cool-down, mainly due to the mismatch between the coefficients of thermal expansion (CTE) of the fibre and matrix. Residual stresses have important effects on the thermo-mechanical behaviour of composite materials and, moreover, the resulting stresses are sufficient to initiate fracture within the matrix immediately around the fibre $[1,2]$. Therefore, it is important to determine the current state of the residual stresses and their effects on the behaviour of the composite when subsequently subjected to multi-axial mechanical loading. After curing and cooling of the composite, the matrix is subject to a tri-axial residual stress state [3]. The resulting thermal residual stresses are of compressive nature in the fibre and tensile nature in the matrix [4]. For glass fibre epoxy resin composites, Fiedler et al. [4] showed that the thermal residual stresses can be calculated by finite element analysis (FEA) using the actual temperature dependent stiffness of the resin [4]. Asp et al. [5] showed by a FEA study that the thermal residual stress strongly reduces the ultimate strength of transversely loaded unidirectional (UD) composites. When load is applied to the fibre-reinforced composite the tri-axial stresses in the matrix increase. Both the polymer matrix and the fibres cannot behave as they would individually as bulk materials, and the difference in the Poisson's ratios causes a tri-axial stress state reducing the maximum bearable load. In recent studies [5-7], it was shown that yield criteria are applicable to glassy polymers under uniaxial, biaxial and triaxial load-

*Corresponding author, e-mail: angelo.maligno@nottingham.ac.uk (C) BME-PT and GTE 
ing if the hydrostatic stress effect is accounted for. Also it was found that for UD composites, yielding is suppressed while a brittle failure due to crack growth occurs. Fiedler et al. [4] also demonstrated that the parabolic Mohr failure criterion is suitable to describe the experimentally observed macroscopic yield and fracture behaviour of epoxy resin $[8,9]$. The micro-residual stresses depending on the local fibre distribution can improve or reduce the local ultimate transverse strength of the composite [10]. In order to clarify the role of thermal residual stresses in composites a number of analytical models have been proposed. Analytical approaches include methods based on the Self-Consistent Model (SCM) of Hill [11], extension of the Eshelby's equivalent inclusion technique [12], Vanishing Fibre Diameter (VFD) model of Dvorak [13], Concentric Cylinder Models [14, 15] and Aboudi's Method of Cells [16]. The effects of thermal residual stresses on mechanical behaviour of the composite materials have also been extensively studied by Nimmer [17] and Wisnom [18]. They examined the transverse behaviour of high temperature composites in the presence of thermally induced residual stress fields and found that the presence of residual stresses is beneficial for the transverse behaviour of composites with low interfacial strength due to the generation of compressive residual stresses at the interface of the fibre and matrix. Three dimensional finite element models have been employed to study the influence of residual stresses on shear response of the composites $[19,20]$. More recently, a finite element micromechanical based model has been developed to investigate the off-axis behaviour of unidirectional composites [21]. This model is general and can be used for any combination of normal and shear loading with residual stresses. Use of FEA with periodic representative volume elements (RVE) is well established [5, 6, 22, 23]. In most cases, the analysis is based on a uniform, square or hexagonal fibre array. In this work, a 3D finite element analysis was used to study the residual stress distribution and its effect on transverse and longitudinal failure and damage evolution of fibre-reinforced polymer matrix composites using a micromechanical RVE model. Moreover, four different fibre volume fraction $\left(V_{f}\right)$ RVEs were investigated in order to evaluate their response to uniaxial loading with and without residual stress. The residual stress intro- duced during curing was determined by considering the contributions from both the chemical shrinkage of resin and the thermal cooling contraction of fibre and resin. Effects of residual stress on damage evolution and failure in RVEs subjected to mechanical loading were predicted using two different failure criteria and a post-failure stiffness reduction technique.

\section{Finite element modelling}

\subsection{Micromechanical model}

Composite materials properties, e.g. strength and stiffness, are dependent upon the fibre volume fraction and individual properties of the constituent fibre and matrix materials and the estimation of damage and failure progression is more complex than in conventional metallic materials. In the micromechanical approach, the constituent fibre and matrix materials and their interaction are distinctively considered to predict the overall behaviour of the composite material structure. The advantage of the micromechanical model is that the stresses can be associated and related to each constituent (fibre and matrix). Therefore, failure can be identified in each of these constituents and the appropriate property degradation can be modelled. Also, different fibre volume fractions can be taken into account by varying the geometry of the RVE. Here, the micromechanical model considers a RVE in which fibre and matrix are assumed to be perfectly bonded to the fibres throughout the analysis, with fibres arranged in a hexagonal cross section array by assuming the repetitive or periodic nature of the fibre and matrix materials. The RVE is a three-dimensional solid and the geometry of each

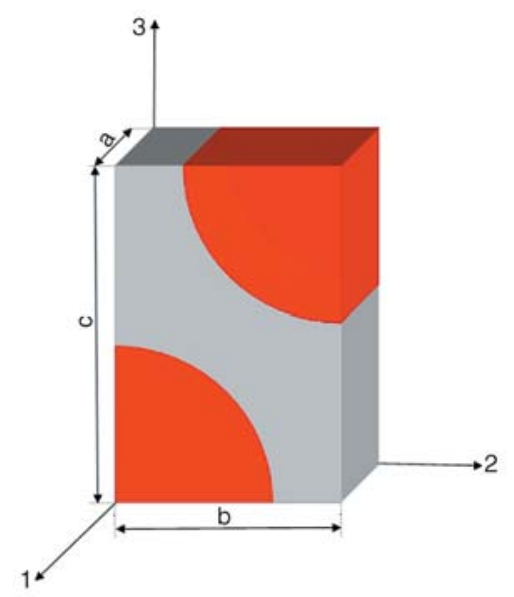

Figure 1. Ideal RVE for the hexagonal array packing 


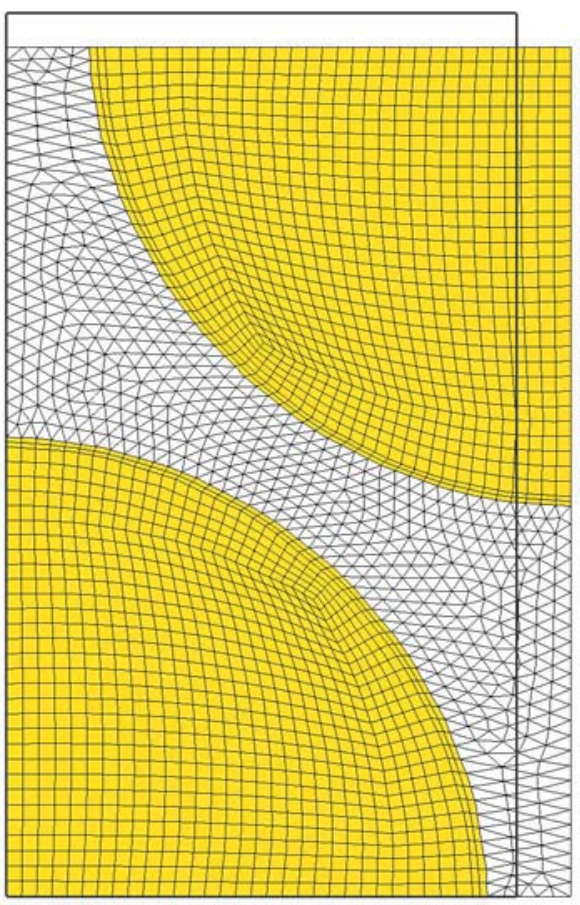

a)

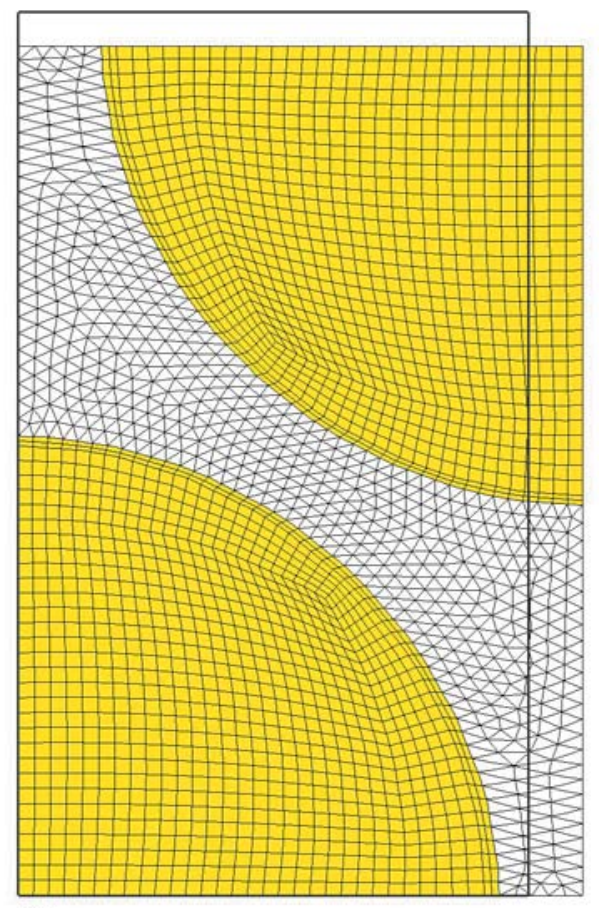

b)

Figure 2. Un-deformed meshed (a) and its deformed shape (b) on transverse loading (2-direction) for an RVE with $V_{f}=70 \%$

RVE depends on the fibre volume fraction [22]. The RVE used in these investigations is displayed in Figure 1. The displacement constraints applied to the finite element model displayed in Figure 1 are [22]: $\mathbf{u}_{\mathbf{1}}(0,2,3)=0 ; \mathbf{u}_{\mathbf{1}}(a, 2,3)=$ constant $=\delta_{1}$; $\mathbf{u}_{2}(1,0,3)=0 ; \mathbf{u}_{2}(1, b, 3)=$ constant $=\delta_{2} ; \mathbf{u}_{3}(1,2,0)=$ $0 ; \mathbf{u}_{3}(1,2, c)=$ constant $=\delta_{3} ;$ where $\mathbf{u}_{1}, \mathbf{u}_{2}, \mathbf{u}_{3}$ denote displacements in the 1-, 2- and 3-direction, respectively. The meshes generated for the micromodels investigated are 20-noded hexahedral elements. The number of elements varies approximately from 6000 to 9000 depending on the $V_{f}$. Mesh sensitivity analysis suggests that the meshes are fine enough to produce accurate results compared to a mesh with twice as many elements, with a difference within $0.2 \%$ in terms of residual stress and failure strain level. In Figure 2 is depicted an example of the mesh for an RVE with a $V_{f}=70 \%$ loaded in the transverse direction (2-direction).

\subsection{Residual stress analysis}

The total induced strain of the resin due to chemical shrinkage and thermal cooling can be expressed by Equation (1):

$$
\mathrm{d} \varepsilon_{i j}=\mathrm{d} e_{i j}+\delta_{i j} \mathrm{~d} s+\delta_{i j} \alpha(T) \mathrm{d} T
$$

where $\mathrm{d} \varepsilon_{i j}$ is the total strain increment, $\mathrm{d} e_{i j}$ the elastic strain increment, $\mathrm{d} s$ the free shrinkage strain increment due to the chemical reaction (cross-linking) in the absence of constraint, $\alpha(T)$ the thermal expansion coefficient which is dependent on the temperature, $\mathrm{d} T$ the temperature change and $\delta_{i j}$ is the Kronecker delta. From Equation (1), the stressstrain relationship can be derived as shown in Equation (2):

$$
\mathrm{d} \sigma_{i j}=C_{i j k l} \mathrm{~d} e_{k l}=C_{i j k l}\left\{\mathrm{~d} \varepsilon_{k l}-\delta_{i j} \mathrm{~d} s-\delta_{i j} \alpha(T) \mathrm{d} T\right\}
$$

where $\mathrm{d}_{i j}$ are the stress increments and $C_{i j k l}$ the stiffness components. The above stress analysis is based on linear elasticity and the stiffness components $C_{i j k l}$ are related to the Young's modulus $E$ and the Poisson's ratio $v$ of the material. Equation (2) was derived for the residual stress analysis in the resin.

\subsection{Material}

Several authors such as Zhao et al. [23] have employed in their studies more realistic constitutive theories for the epoxy matrix (e.g. non linear viscoelasticity). However, numerical results have shown that damage onset and evolution are not 
influenced by the stress-relaxation, induced by the viscoelastic behaviour and, the final amount of residual stress (after cooling) is not in general significantly affected by the viscoelastic property of the resin. Hence, residual stress and its effect on transverse and longitudinal failure of UD composites have been investigated considering the linearelastic behaviour of the constituents. In particular, the materials used in this investigation are glass fibre and epoxy resin, whose properties are given in [24]. The properties of glass fibre are assumed to remain constant and independent of the temperature change with Young's modulus $E=80 \mathrm{GPa}$ and Poisson's ratio $v=0.22$, the coefficient of thermal expansion $\alpha=4.9 \cdot 10^{-6} /{ }^{\circ} \mathrm{C}$ and the longitudinal tensile $\left(\sigma_{T}\right)$ and compressive $\left(\sigma_{C}\right)$ strength are 2150 and $1450 \mathrm{MPa}$, respectively. However, for the epoxy resin, thermal transition temperatures such as the glass transition temperature $T_{g}$ strongly affect mechanical properties [24]. In order to represent this behaviour accurately the material properties of the resin are defined as a function of temperature. The following relations are used:

(a) Poisson's ratio is assumed to be temperature independent $(v=0.35)$.

(b) To evaluate the variation of Young's modulus $E$ over the temperature range from curing to room temperature, the total temperature range can be divided into three regions:

$-T_{g}-\Delta T \leq T \leq T_{g}+\Delta T$, in which $E$ varies greatly.

$-T>T_{g}+\Delta T$, the matrix is in liquid or rubbery state and $E$ has a very small value.

$-T<T_{g}-\Delta T$, the matrix is in solid state and $E$ changes only slightly.

For each region, the modulus is obtained using the following functions given by Equations (3)-(5) [25]:

$$
E(T)=E\left(T_{r}\right) \exp \left(-k_{1} \frac{T-T_{r}}{T_{g}-\Delta T-T_{r}}\right),
$$

$T<T_{g}-\Delta T$,

$E(T)=E\left(T_{g}-\Delta T\right) \exp \left(-k_{2} \frac{T-T_{g}+\Delta T}{\Delta T+\Delta T}\right)$,

$T_{g}-\Delta T \leq T \leq T_{g}+\Delta T$,

$E(T)=0.01 E\left(T_{r}\right), T>T_{g}+\Delta T$, with: $T_{g}=110^{\circ} \mathrm{C} ; T_{r}=23^{\circ} \mathrm{C} ; \Delta T=35^{\circ} \mathrm{C} ; E\left(T_{r}\right)=$ $3.35 \mathrm{GPa} ; \quad E\left(T_{g}-\Delta T\right)=0.7 E\left(T_{r}\right) ; \quad E\left(T_{g}+\Delta T\right)=$ $0.01 E\left(T_{r}\right) ; k_{1}=0.357 ; k_{2}=4.249$.

(c) The thermal expansion coefficient $\alpha$ is assumed to change linearly with the temperature: $\alpha(T)=$ $K\left(T-T_{r e f}\right)+\alpha\left(T_{r}\right)$, with a slope given by Equation (6):

$$
K=\frac{\alpha_{l}-\alpha\left(T_{r}\right)}{T_{g}-T_{r}}
$$

where $\alpha\left(T_{r}\right)=58 \cdot 10^{-6} /{ }^{\circ} \mathrm{C}$ and $\alpha_{l}=139 \cdot 10^{-6} /{ }^{\circ} \mathrm{C}$.

The longitudinal tensile $\left(\sigma_{T}\right)$ and compressive $\left(\sigma_{C}\right)$ strength of the resin are taken to be 80 and $120 \mathrm{M} \mathrm{Pa}$, respectively.

\section{Failure criteria and damage evolution model}

The selection of a proper failure criterion, both for matrix and fibre, represents a very important task of the modelling formulation. In particular in polymers the yield behaviour is sensitive to hydrostatic stress and as a consequence, the yield stress in tension is different from that in compression [7, 26, and 27]. Both fibre and resin are isotropic materials and the Maximum Principal Stress theory is applicable to simulate damage onset and evolution within the RVE (e.g. fibre/matrix debonding, matrix crack). If the stress level satisfies the failure criterion, the fibre or matrix would crack. Final failure corresponds to the rupture of the composite, which is unable to carry further load. The Maximum Principal Stress failure criterion is summarized as shown in Equations (7) and (8):

$$
\begin{gathered}
\sigma_{\max }<\sigma_{u}^{t} \\
\left|\sigma_{\min }\right|<\sigma_{u}^{c}
\end{gathered}
$$

where $\sigma_{\max }$ and $\sigma_{\min }$ are the Maximum and Minimum Principal Stresses, $\sigma_{u}^{t}$ is the tensile strength and $\sigma_{u}^{c}$ is the compressive strength of the material. A modification of the von Mises criterion has also been also considered to evaluate failure in the matrix. As the von Mises criterion does not predict differences in yield stress between tension and compression, modifications of this criterion have incorporated the effects of hydrostatic pressure. A general form for the modified von Mises criterion can be written as shown in Equation (9) [7]: 


$$
\begin{aligned}
& A\left(\sigma_{1}+\sigma_{2}+\sigma_{3}\right)+ \\
& B\left[\left(\sigma_{1}-\sigma_{2}\right)^{2}+\left(\sigma_{2}-\sigma_{3}\right)^{2}+\left(\sigma_{3}-\sigma_{1}\right)^{2}\right]=1
\end{aligned}
$$

It is possible to determine the constants $A$ and $B$ in term of the simple uniaxial tensile $\left(\sigma_{y, T}\right)$ and compressive $\left(\sigma_{y, C}\right)$ yield stresses. The modified von Mises stress criterion was suggested by Raghava and has been shown to agree very well with experimental data for epoxy resin - see Equation (10) [7]:

$$
\begin{aligned}
& 2\left(\sigma_{y, C}-\sigma_{y, T}\right)\left(\sigma_{1}+\sigma_{2}+\sigma_{3}\right)+ \\
& {\left[\left(\sigma_{1}-\sigma_{2}\right)^{2}+\left(\sigma_{2}-\sigma_{3}\right)^{2}+\left(\sigma_{3}-\sigma_{1}\right)^{2}\right]=2 \sigma_{y, C} \sigma_{y, T}}
\end{aligned}
$$

If $\sigma_{y, C}=\sigma_{y, T}$ this criterion reduces to the von Mises criterion. To simulate damage, it is necessary to evaluate the current stress state at each integration point. Then by comparing the current stress state with a specific failure criterion, the material properties are reduced at each 'failed' integration point to values representing the particular type of damage that has occurred [28-30]. The degradation scheme, together with the residual stress analysis was programmed into a user-defined material subroutine (UMAT) interfaced with the commercial finite element code ABAQUS Standard [31]. When failure is detected the degradation is applied only on the elastic moduli by multiplying them with a discount factor $d_{i} \in(0,1]$ ( $i$ designates the elastic modulus to which the factor is applied). Both resin and fibre were modelled as isotropic with they the stiffness matrix shown in Equation (11):

\section{$[C]=[S]^{-1}=$}

$$
\left[\begin{array}{cccccc}
\frac{1}{d_{E} E} & -\frac{v}{d_{E} E} & -\frac{v}{d_{E} E} & 0 & 0 & 0 \\
-\frac{v}{d_{E} E} & \frac{1}{d_{E} E} & -\frac{v}{d_{E} E} & 0 & 0 & 0 \\
-\frac{v}{d_{E} E} & -\frac{v}{d_{E} E} & \frac{1}{d_{E} E} & 0 & 0 & 0 \\
0 & 0 & 0 & \frac{1}{d_{G} D} & 0 & 0 \\
0 & 0 & 0 & 0 & \frac{1}{d_{G} D} & 0 \\
0 & 0 & 0 & 0 & 0 & \frac{1}{d_{G} D}
\end{array}\right]^{-1}
$$

The Young's modulus $E$ and the shear modulus $G$ are degraded independently by discount factor $d_{E}$ and $d_{G}$ both initially set equal to the unity. If during the analysis the stress level exceed the maximum strength allowed for matrix and/or fibre according to the failure criterion, the modulus $E$ is degraded to $1 \%$ of its initial value $\left(d_{E}=0.01\right)$ at the particular integration point. The shear modulus $G$ is reduced to $20 \%$ of the initial value $\left(d_{G}=0.2\right)$ under the assumption that some shear stiffness remains due to the friction still present on the failure plane [30]. The behaviour of the matrix and the fibre was assumed to be linear elastic until damage was predicted. The response after damage occurred was also linear elastic but with degraded moduli.

\section{Results and discussion}

\subsection{Residual stress}

Residual stress has two parts: the chemical shrinkage residual stress and the thermal cooling residual stress. The analysis was performed by two discrete steps, where step one is the shrinkage stress analysis and step two is the thermal cooling stress analysis. The shrinkage residual stress was calculated by applying a given amount of resin shrinkage. For the epoxy resin considered here, the linear shrinkage strain was chosen to be $1 \%$, which corresponds to a volumetric change of less than 3\% [32] depending on the fibre volume fraction and the effect of the fibres on longitudinal shrinkage during curing. The thermal residual stress is due to the cooling of the system from the curing temperature, $149^{\circ} \mathrm{C}$, to room temperature, $23^{\circ} \mathrm{C}$. The distribution of the resin's maximum principal residual stress in the matrix, after curing and cooling is presented in Figure 3. The mechanical properties of the resin, in terms of shear modulus (and Young's modulus

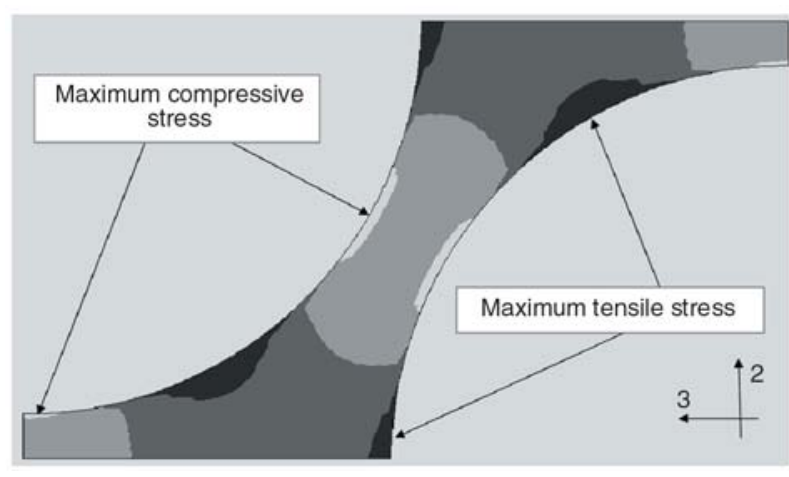

Figure 3. Distribution of residual stress in the matrix after curing and cooling-down 


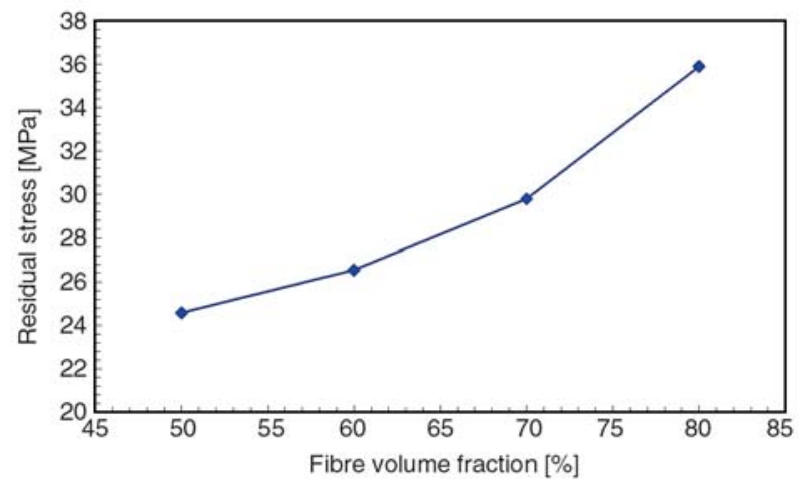

Figure 4. Trend of maximum principal residual stress [MPa] after curing and cooling-down evaluated in the area of max tensile stress at different $V_{f}$

when the resin becomes solid), increase drastically as the material evolves from a liquid state to a solid state. So in the resin, geometrically constrained within the interstices present between fibres, tensile stresses develop more easily. The largest values reached after chemical shrinkage and cooling are depicted in Figure 4. Results from the analysis attribute the primary contribution to residual stress mainly to thermal cooling. In Figure 5 the small contribution from the chemical shrinkage for four different fibre volume fractions is shown. Moreover, as afore mentioned, the small calculated internal stress level, due to the curing process, is likely to be overestimated since the viscoelastic properties of the resin are not taken into account (i.e. these stresses are likely to relax further with time) [23]. These curing stresses are small as a result of the mechanical properties of the resin during the curing process. In fact, its state is rubbery and almost liquid so the capability to interact with the fibres by transferring stresses is negligible. These results agree with most of the published work on residual

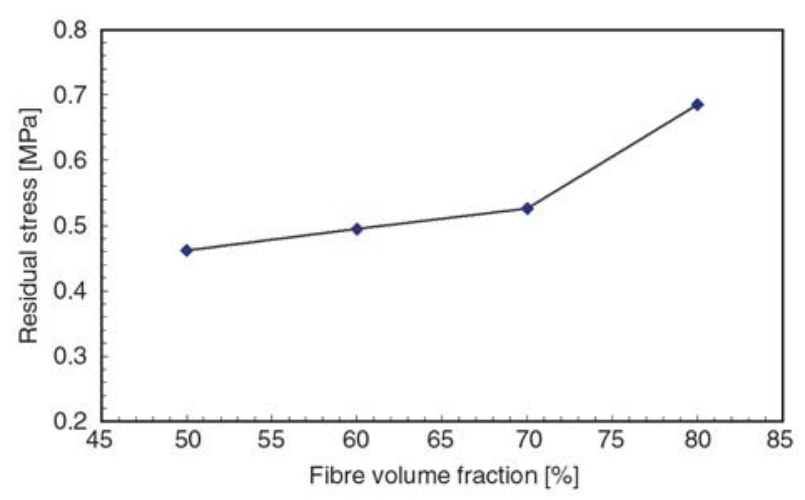

Figure 5. Trend of maximum principal residual stress $[\mathrm{MPa}]$ after curing evaluated in the area of max tensile stress at different $V_{f}$ stresses in which thermal cooling was evaluated as the main source of residual stress in polymer composites [33, 34].

\subsection{Effect of residual stress on transverse and longitudinal failure}

To study the influence of residual stress/strain on the overall response of the composites at their microscale, the damage evolution in the matrix was examined under transverse loading. After curing and thermal cooling analyses, a global strain was applied to the micro-models which were achieved by specifying a uniform displacement on the RVEs faces. At each time increment of the analysis, the damaged area in the matrix was determined using both the Maximum Principal Stress failure criterion and the von Mises criterion modified by Raghava. Throughout the following analyses, the fibre showed no sign of damage due to its high strength, therefore, damage and failure refer to the matrix only.

\subsubsection{Uniaxial tensile loading along 1-direction}

In UD composites the effect of the fibre is dominant, therefore during curing, in which resin is in a rubbery state, realistically, no deformation is established along the 1-direction. The strains, developed during the manufacturing process, are parallel to the fibres orientation as the resin evolves from a liquid/rubbery state into a solid state. In addition, as the fibres are dominant, the strength of the micromodels is improved if fibre volume fraction is increased. A comparison between two different combinations of failure criteria was investigated:

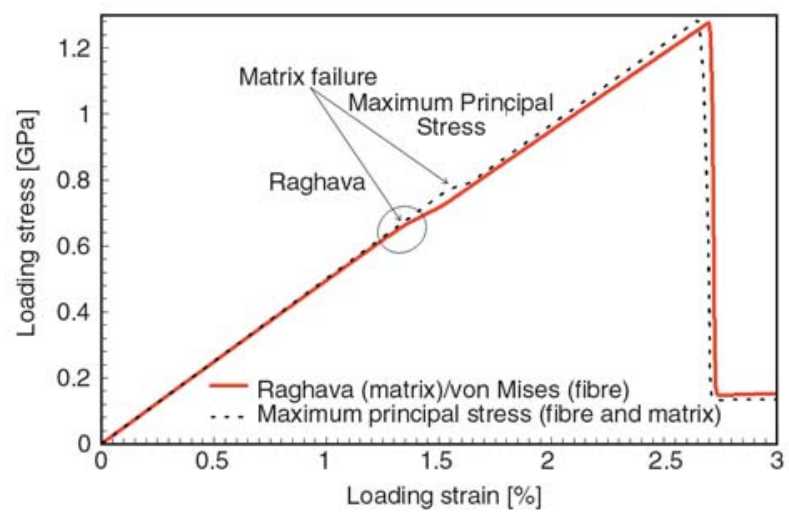

Figure 6. Global stress-strain curve in 1-direction for uniaxial tensile loading for $V_{f}=60 \%$ (Residual stress analysis) 


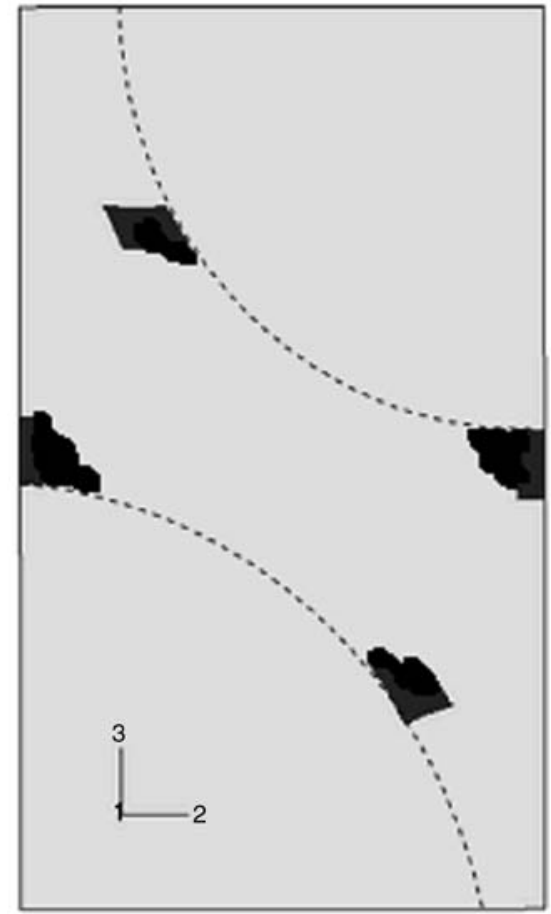

a)

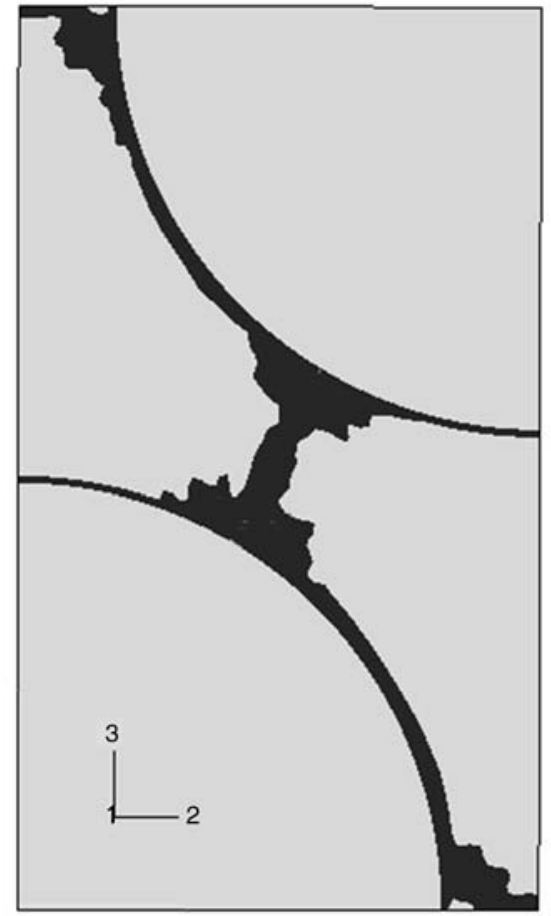

b)

Figure 7. Comparison on damage onset prediction $\left(V_{f}=60 \%\right)$ in presence of residual stress. Maximum Principal Stress (a), Raghava failure criterion (b).

1 - Raghava (matrix)/von Mises (fibre).

2 - Maximum Principal Stress (matrix)/Maximum Principal Stress (fibre).

Both combinations show similar results in terms of ultimate strength for the fibre with residual stress (Figure 6) but, the Raghava criterion predicts matrix failure at a lower loading strain. In addition, the two combinations of failure criteria show a difference in the prediction of damage onset in the matrix. In fact, while for the Maximum Principal Stress the initiation of the damage is concentrated in four regions at the fibre/matrix interface (Figure 7a), the Raghava failure criterion predicts a drastic fibre/matrix debonding and also damage within the resin at the interstices between fibres, as shown in (Figure 7b). Comparison between stress/ strain curves with and without residual stress (Figure 8) shows that a premature matrix failure occurs if residual stresses are applied. In fact, it is evident from Figure 8 that, although the presence of thermal residual stresses does not modify the ultimate strength of fibres along the 1-direction of loading, it leads to a greatly detrimental reduction in the epoxy matrix on its capability to bear loads. Residual stresses are always detrimental for the matrix in 1-direction longitudinal loading and in the case of biaxial (especially 1-2, 1-3 direction) or even triax-

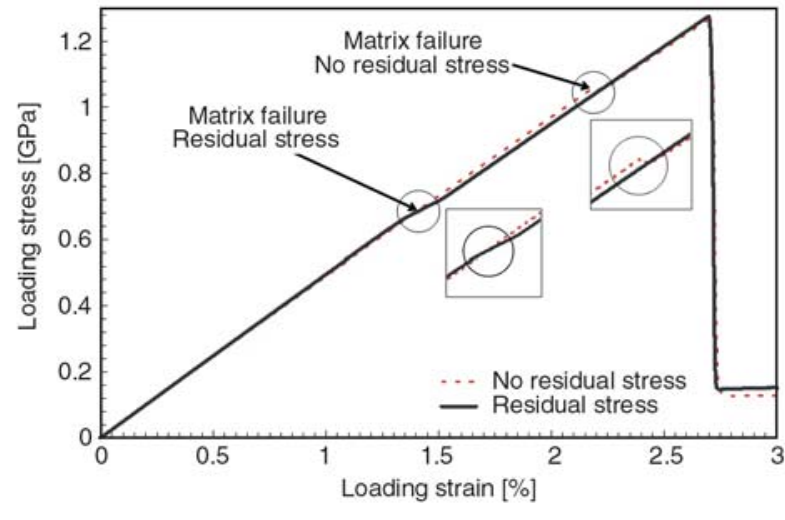

Figure 8. Global stress-strain curve in 1-direction for uniaxial tensile loading for $V_{f}=60 \%$. Failure criteria: von Mises for the fibre and Raghava for the matrix.

ial states of loading small loading strains could cause debonding at the fibre/matrix interface.

\subsubsection{Uniaxial tensile loading along 2-direction and 3-direction}

On transverse uniaxial tensile loading along the 2direction, the Maximum Principal Stress criterion predicts the damage initiation (represented by black shading) to start from the corners of the RVE (Figure 9a), while the failure propagates within the matrix along the edges of the micro-model (Fig- 


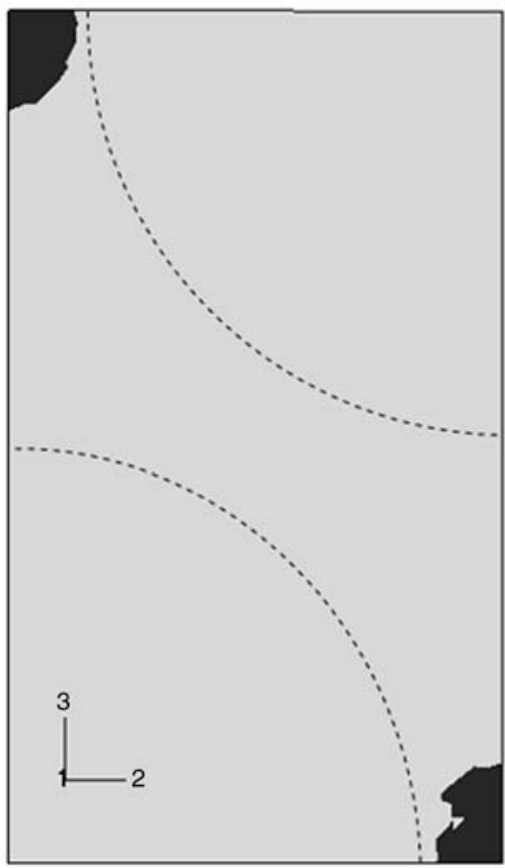

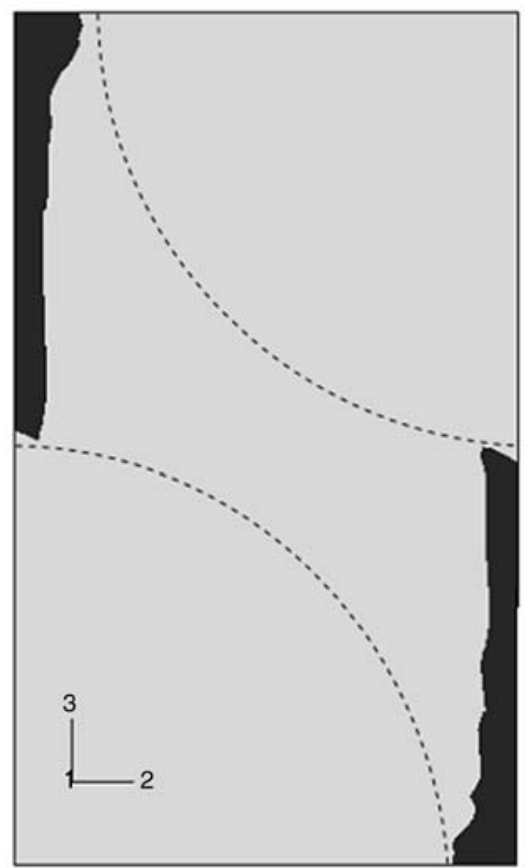

b)

Figure 9. Damage initiation (a) and evolution (b) under uniaxial transverse tensile loading along 2-direction evaluated by means of the Maximum Principal Stress with no residual stress $\left(V_{f}=60 \%\right)$

ure $9 \mathrm{~b}$ ). The evolution of damage for uniaxial tensile loading in the 2-direction in the residual stress analysis is shown in Figure 10. The residual stress/ strain state corresponds to the conditions of $1 \%$ shrinkage strain and $149^{\circ} \mathrm{C}$ curing temperature. It can be seen that the site of damage initiation and the subsequent evolution are clearly affected by thermal residual stress. In fact, FE analysis proved that the damage initiates at the fibre/matrix interface (Figure 10a) and evolves along the fibre/ matrix interface (Figure 10b). The damage onset in the 3-direction with no residual stress takes place at the fibre/matrix interface as depicted in Figure 11. For this load case damage initiation and its evolu-

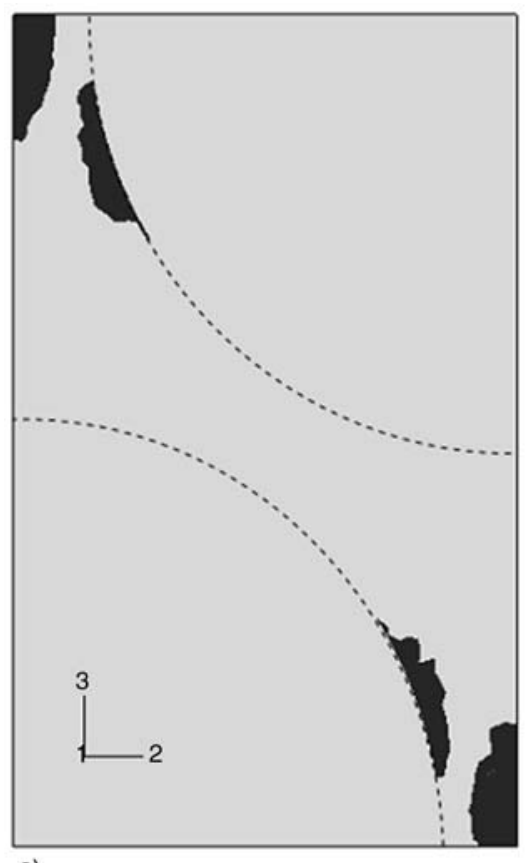

a)

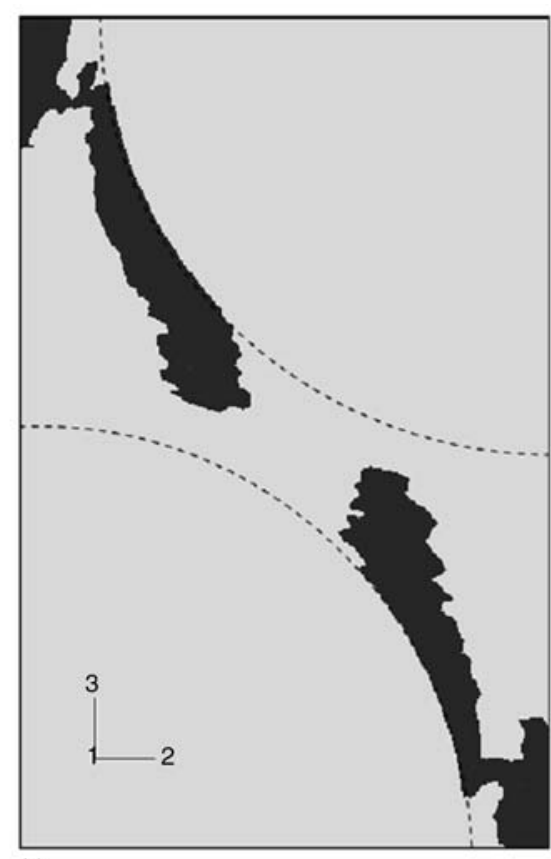

b)

Figure 10. Damage initiation (a) and evolution (b) under uniaxial transverse tensile loading along 2-direction evaluated by means of the Maximum Principal Stress with residual stress $\left(V_{f}=60 \%\right)$ 

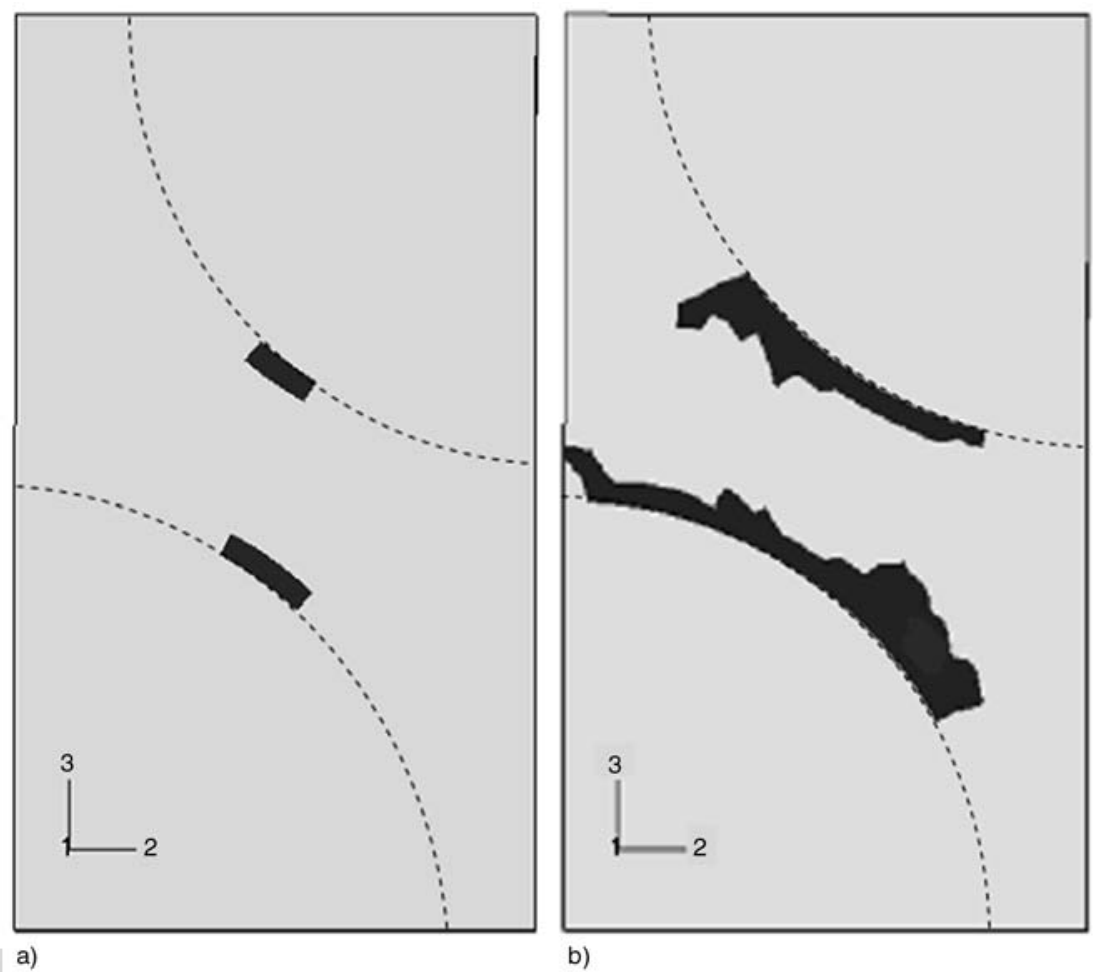

Figure 11. Damage initiation (a) and evolution (b) under uniaxial transverse tensile loading along 3-direction evaluated by means of Maximum Principal Stress criterion with residual stress $\left(V_{f}=60 \%\right)$

tion are not affected significantly by the presence of residual stresses. A crucial result is that the initiation of the damage depends on the mode of loading: 2-direction or 3-direction. As displayed in Figure 9a and Figure 11a the damage onset occur within the matrix in different areas. Specifically, in the 2-direction, damage takes place in the corners of the RVE and in the 3-direction, at the fibre/ matrix interface in the centre of the RVE. The interpretation for such a difference is due to the particular line of symmetry [35] in the RVE under investigation in which fibres are assembled with a hexagonal packing array (Figure 12). Hence the stress field, distributed symmetrically about the line of

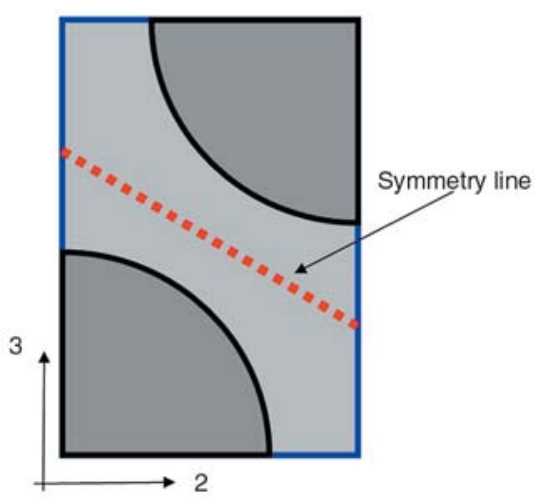

Figure 12. Line of symmetry in a RVE (hexagonal packing array) symmetry, is different than in a square packing array. During the damage analysis, the global stress-strain response in the loading direction was monitored and an example of results is given in Figure 13 for the cases with and without residual stress. For both cases, the carried stress starts to drop from the point of damage initiation. Once damage is initiated, the model tends to fail suddenly. Thus, the initial failure strain level is also the final failure level for transverse loading. This brittle behaviour is also observed under the 3-direction loading. In Figure 14 the dependence of ultimate strength on fibre content is displayed for these load directions in the case of no residual stress. The

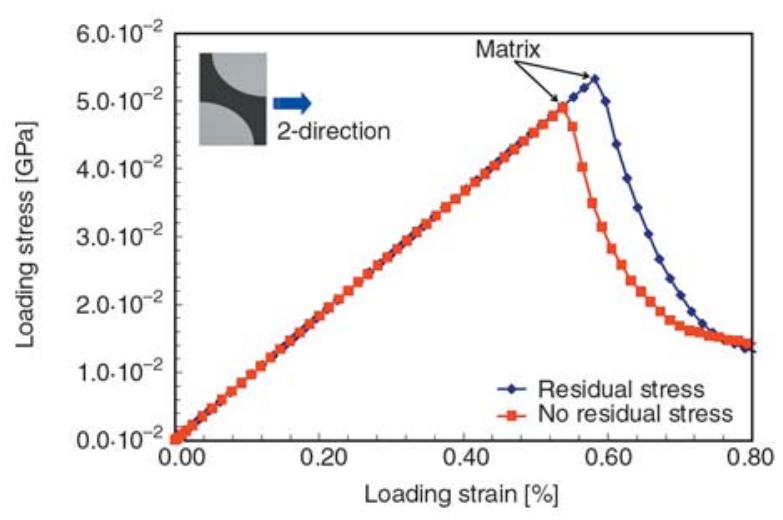

Figure 13. Global stress-strain curve in 2-direction for uniaxial transverse tensile loading 


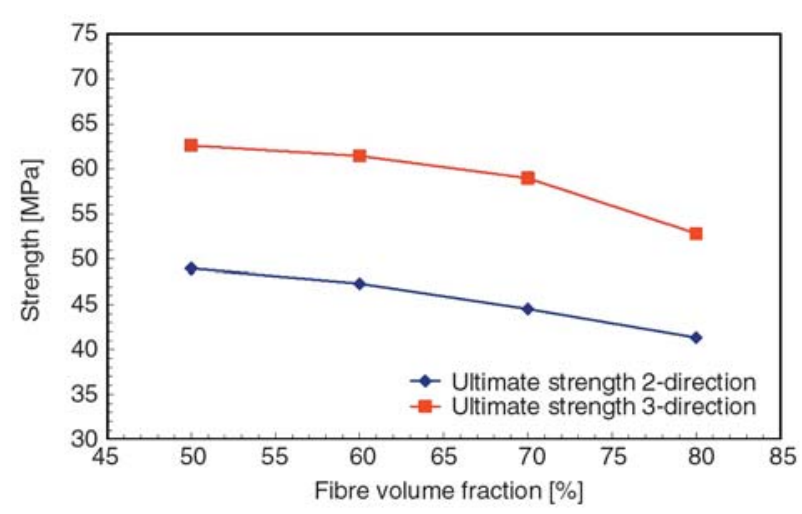

Figure 14. Comparison of ultimate strength for 2-direction and 3-direction loading by using the Maximum Principal Stress criterion. No residual stress applied.

comparison between the strengths predictions using the Maximum Principal Stress and the Raghava failure criteria at different fibre content has an immediate consequence. In fact, the Raghava failure criterion shows the same location in predicting damage onset for the all cases (no-residual stress and residual stress, 2- and 3-direction loading state)

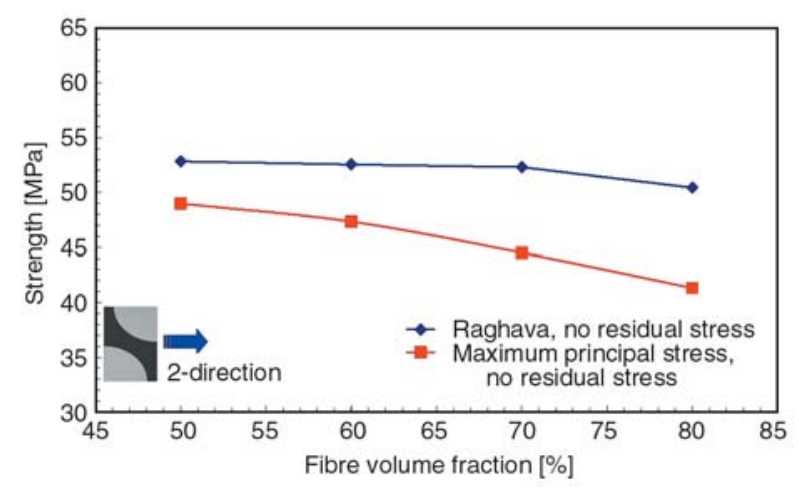

Figure 15. Trend of ultimate strength $[\mathrm{MPa}]$ in 2-direction evaluated with Maximum Principal Stress and Raghava failure criteria. No residual stress.

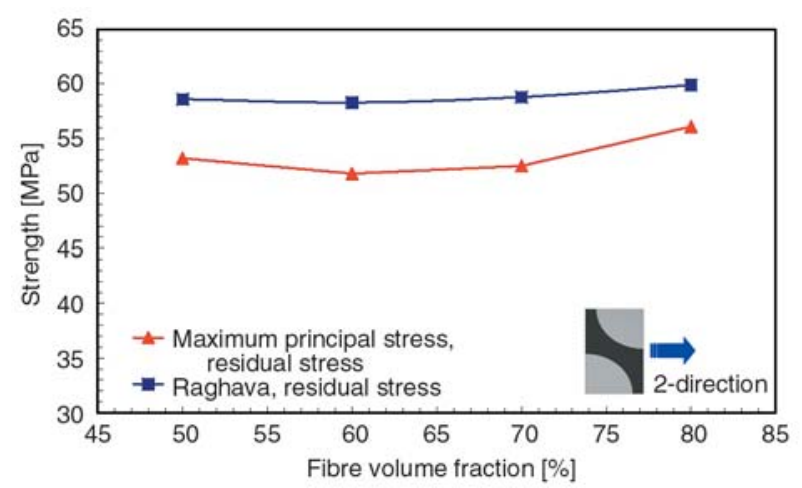

Figure 16. Trend of ultimate strength $[\mathrm{MPa}]$ in 2-direction evaluated with Maximum Principal Stress and Raghava failure criteria. Effect of residual stress.

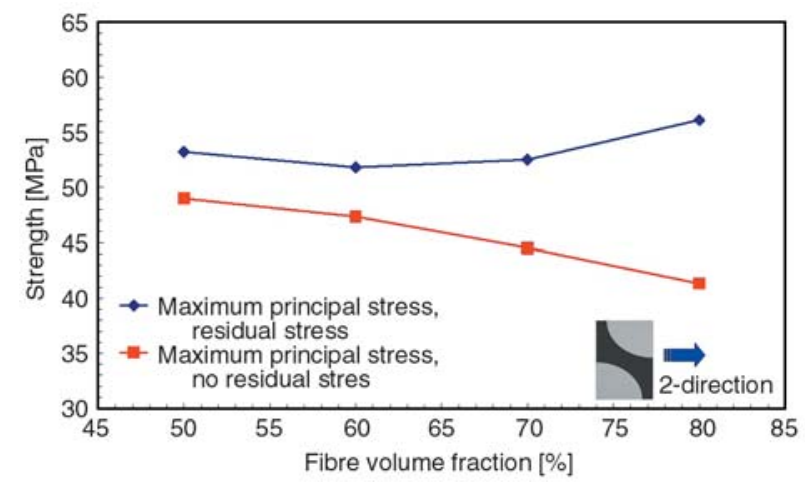

Figure 17. Ultimate strength $[\mathrm{MPa}]$ with and without residual stress (2-direction)

but it is always less conservative in terms of ultimate strength (Figure 15 and Figure 16). Furthermore, the presence of residual stress in the 2-direction (Figure 17) and 3-direction loading is in general beneficial. Residual stresses, arising during the simulated manufacturing process, imply a redistribution of the internal stress field which in general leads to lower stresses within the resin and, to improve the ability to bear loads in the transverse directions. Numerical models with a low fibre volume fractions undergo a detrimental effect due to thermal residual stress when the model is loaded in the 3-direction. After cooling the highest values of residual stresses in the matrix are concentrated along the fibre/matrix interface in particular areas but the evolution of the stress field by applying a displacement in 3-direction is dissimilar. In fact, for high fibre volume fractions stresses tend to spread internally within the resin whilst for low fibre contents they remain highly concentrated at the interface throughout the analysis, weakening this area significantly. Hence, residual stresses could play an important role in decreasing the overall response of the composite negating the potential beneficial effects for 2- and 3-direction transverse loading and producing a drastic failure of the composite.

\section{Conclusions}

Residual stress and its effect on transverse and longitudinal failure of UD glass fibre/epoxy resin composites were studied using a micromechanical RVE model and the finite element methods. The overall residual stress is determined by considering two contributions: volume shrinkage of matrix resin from the crosslink polymerization during 
isothermal curing and thermal contraction of both resin and fibre as a result of cooling from the curing temperature to room temperature. Analyses confirm the isothermal residual stress during curing can be considered negligible compared to the thermal residual stress due to cooling. A study of two different failure criteria was also performed for the epoxy matrix in order to evaluate differences in the capability to predict failure. The assessment of these failure criteria has proved their capability to describe qualitatively the material behaviour of the UD composites under longitudinal and transverse loading. Interestingly, energy based failure criteria (e.g. Raghava), also demonstrated in [36], are particular sensitive to high triaxial stresses which arise at the fibre/matrix interface on uniaxial tensile loading parallel to the fibres orientation and, due to the particular assumptions applied to the RVEs (e.g. perfect bonding between fibres and matrix) in these numerical investigations. Numerical analyses have shown that predicted damage initiation and evolution are clearly influenced by the presence of residual stress. In particular, residual stress causes a premature failure in the matrix at a lower strain than with no residual stress conditions on longitudinal loading case (1-direction) and it is always detrimental for the matrix while for the fibre there is no important alteration in terms of ultimate strength. In addition, the effect of residual stress on transverse tensile loading (2- and 3-direction) depends on the fibre volume fraction and produces beneficial results in the 2-direction at the fibre volume fractions studied while, in 3-direction it is detrimental for low fibre volume fractions.

\section{Acknowledgements}

This work is funded by the UK Engineering and Physical Sciences Research Council (EPSRC) through the Nottingham Innovative Manufacturing Research Centre (NIMRC).

\section{References}

[1] Zahl D. B., McMeeking M. R.: The influence of residual stress on the yielding of metal matrix composites. Acta Metallurgica et Materialia, 39, 1117-1122 (1991).

[2] Bigelow C. A.: Thermal residual stresses in a siliconcarbide/titanium [0/90] laminate. Journal of Composites Technology and Research, 15, 304-310 (1993).
[3] Hull D., Clyne T. W.: An introduction to composite materials. Cambridge University Press, Cambridge (1996).

[4] Fiedler B., Hojo M., Ochiai S.: The influence of thermal residual stresses on the transverse strength of CFRP using FEM. Composites Part A: Applied Science and Manufacturing, 33, 1323-1326 (2002).

[5] Asp L. E., Berglund L. A., Talreja R.: Prediction of matrix-initiated transverse failure in polymer composites. Composites Science and Technology, 56, 10891097 (1996).

[6] Asp L. E., Berglund L. A., Gudmundson P.: Effects of a composite-like stress state on the fracture of epoxies. Composites Science and Technology, 53, 27-37 (1995).

[7] Asp L. E., Berglund L. A., Talreja R.: A criterion for crack initiation in glassy polymers subjected to a composite like stress state. Composites Science and Technology, 56, 1291-1301 (1996).

[8] Fiedler B., Hojo M., Ochiai S., Schulte K.: Yield criteria for polymers in composite-like stress state. in 'Proceeding of the 23rd Symposium of the Society of Composite Materials Japan. Yamagata, Japan', 154155 (1998).

[9] Fiedler B., Hojo M., Ochiai S., Schulte K., Ando M.: Failure behavior of an epoxy matrix under different kinds of static loading. Composites Science and Technology, 61, 1615-1624 (2001).

[10] Fiedler B., Hojo M., Ochiai S., Schulte K., Ochi M.: Finite-element modeling of initial matrix failure in CFRP under static transverse tensile load. Composites Science and Technology, 61, 95-105 (2001).

[11] Gramoll K. C., Walker K. P., Freed A. D.: An overview of self-consistent methods for fibre reinforced composites. NASA TM 103713 (1991).

[12] Mori T., Tanaka K.: Average stress in matrix and average elastic energy of materials with misfitting inclusions. Acta Metallurgica, 21, 571-574 (1973).

[13] Yeh N. M., Krempl E.: The influence of cool-down temperature histories on the residual stresses in fibrous metal-matrix composites. Journal of Composite Materials, 27, 973-995 (1993).

[14] Uemura M., Iyama H., Yamaguchi Y.: Thermal residual stresses in filament-wound carbon-fibre-reinforced composites. Journal of Thermal Stresses, 2, 393-412 (1979).

[15] Mikata Y., Taya M.: Stress field in a coated continuous fiber composite subjected to thermo-mechanical loadings. Journal of Composite Materials, 19, 554 578 (1985).

[16] Jayaraman K., Reifsnider K. L.: Residual stresses in a composite with continuously varying Young's modulus in the fibre/matrix interphase. Journal of Composite Materials, 26, 770-791 (1992).

[17] Nimmer R. P.: Fibre-matrix interface effects in the presence of thermally induced residual stress. Journal of Composites Technology and Research, 12, 65-75 (1990). 
[18] Wisnom M. R.: Factors affecting the transverse tensile strength of unidirectional continuous silicon carbide fibre reinforced 6061 Aluminium. Journal of Composite Materials, 24, 707-726 (1990).

[19] Nedele M. R., Wisnom M. R.: Finite element micromechanical modelling of a unidirectional composite subjected to axial shear loading. Composites, 25, 263-272 (1994).

[20] Aghdam M. M., Pavier M. J., Smith D. J.: Micromechanical modelling of metal matrix composites subjected to combined thermal and shear loading. in 'Proceedings of the Sixth International Conference on Computer Methods in Composite Materials (CADCOMP 98). Montreal, Canada', 321-330 (1998).

[21] Aghdam M. M., Pavier M. J., Smith D. J.: Micromechanics of off-axis loading of metal matrix composites using finite element analysis. International Journal of Solids and Structures, 38, 3905-3925 (2001).

[22] Sun C. T., Vaidya R. S.: Prediction of composite properties from a representative volume element. Composites Science and Technology, 56, 171-179 (1996).

[23] Zhao L. G., Warrior N. A., Long A. C.: A thermo-viscoelastic analysis of process-induced residual stress in fibre-reinforced polymer-matrix composites. Material Science and Engineering: A. Structural Materials: Properties, Microstructure and Processing, 452, 483498 (2007).

[24] Soden P. D., Hinton M. J., Kaddour A. S.: Lamina properties, lay-up configurations and loading conditions for a range of fibre-reinforced composite laminates. in 'Failure criteria in fibre-reinforced-polymer composites' (eds.: Soden P. D., Hinton M. J., Kaddour A. S.) Elsevier, Oxford, 30-51 (2004).

[25] Zhang Y., Xia Z., Ellyin F.: Evolution and influence of residual stress/strains of fiber reinforced laminates. Composites Science and Technology, 64, 1613-1621 (2004).

[26] Altenbach H., Tushtev K.: A new static failure criterion for isotropic polymers. Mechanics of Composite Materials, 37, 475-482 (2001).
[27] Altenbach H.: A unified phenomenological limit state criterion for ductile and brittle materials. in 'Conference on Plasticity and Impact Mechanics, New Delhi, India' 42-51 (2003).

[28] Tabiei A., Ivanov I.: Materially and geometrically non-linear woven composite micro-mechanical model with failure for finite element simulations. International Journal of Non-Linear Mechanics, 39, 175-188 (2004).

[29] Tabiei A., Song G., Jiang Y.: Strength simulation of woven fabric composite materials with material nonlinearity using micromechanics based model. Journal of Thermoplastic Composite Materials, 16, 5-20 (2003).

[30] Blackketter D. M., Walrath D. E., Hansen A. C.: Modelling damage in a plain weave fabric-reinforced composite material. Journal of Composites Technology and Research 15, 136-142 (1993).

[31] Hibbitt D., Karlsson B., Sorensen P.: ABAQUS v6.5. User's Manuals. Providence (RI) (2005).

[32] Zhang L., Ernst L. J., Brouwer H. R.: Transverse behavior of a unidirectional composite (glass fibre reinforced unsaturated polyester). Part II. Influence of shrinkage strains. Mechanics of Materials, 27, 37-61 (1998).

[33] Eom Y., Boogh L., Michaud V., Sunderland P., Manson J.-A.: A Stress-initiated void formation during cure of a three-dimensionally constrained thermoset resin. Polymer Engineering and Science, 41, 492-503 (2001).

[34] Lange J., Toll S., Manson J.-A. E., Hult A.: Residual stress build-up in thermoset films cured below their ultimate glass transition temperature. Polymer, 38, 809-815 (1997).

[35] Li S.: On the unit cell for micromechanical analysis of fibre-reinforced composites. in 'Proceeding of the Royal Society of London, London, UK', Vol 455, 815-838 (1999).

[36] Maligno A. R.: Finite element investigations on the microstructure of composite materials. PhD Thesis, The University of Nottingham (2008). 